Revista de Psicología Vol. 32 (1), 2014 (ISSN 0254-9247)

\title{
Estilo de vida y presencia de síndrome metabólico en estudiantes universitarios. Diferencias por sexo
}

\author{
María Araceli Álvarez Gasca ${ }^{1}$, María del Rocío Hernández Pozo², \\ Marcela Jiménez Martínez ${ }^{3}$ y Ángel Durán Díaz \\ Universidad Nacional Autónoma de México - México
}

El presente estudio busca analizar la relación entre estilo de vida y el síndrome metabólico en estudiantes universitarios, así como establecer si existen diferencias por sexo. Los participantes fueron 970 estudiantes (67.4\% fueron mujeres y 32.6\% hombres) seleccionados a partir de un muestreo aleatorio. Se evaluó el Estilo de vida (EV), la Obesidad central y el Síndrome metabólico (SM). Los resultados mostraron SM en $4.63 \%$ y obesidad en $36.65 \%$, con mayor frecuencia en las mujeres. El EV predominante fue bueno y mejor en hombres que en mujeres, y la mayor frecuencia del SM fue en el EV malo. La relación entre género, obesidad, y SM fue significativa para la población estudiada. Se encontraron diferencias entre hombres y mujeres.

Palabras clave: Estilo de vida, síndrome metabólico, género, estudiantes universitarios

\section{Lifestyle and metabolic syndrome in college students: Differences by gender}

The relationship between lifestyle and metabolic syndrome in college students as well as differences between men and women are analyzed. 970 students $(67.4 \%$ women, $32.6 \%$ men) were randomly selected and assessed on lifestyle (EV), central obesity, and metabolic syndrome(SM). Results showed 4.63\% with SM and 36.65\% with obesity, women predominated. Predominant EV was good and better in men than women, highest frequency of SM was in bad EV. Relationship between gender, obesity, and SM was significant for the studied population. Differences were found between men and women.

Keywords: lifestyle, metabolic syndrome, gender, college students

1 Magíster en Modificación de Conducta, Profesora titular en la carrera de Medicina en la Universidad Autónoma de México. Dirección postal: Parque del Contador 5, Jardines del Alba, Cuautitlán Izcalli, Estado de México, C.P. 54750, México. Contacto: maragasca@gmail.com

2 Doctora en Psicología, Profesora titular en el posgrado de Psicología en la Universidad Autónoma de México. Contacto: herpoz@unam.mx

3 Licenciada en Medicina por el Instituto Politécnico Nacional. Profesora asociada en la carrera de Medicina en la Universidad Autónoma de México. Contacto: marcelasofia62@gmail.com

4 Magíster en Estadística, Profesor titular en la Carrera de Biología y Asesor de estadística en proyectos de investigación en la Universidad Autónoma de México. Contacto:angeldurandiaz@ gmail.com 
Los estilos de vida o la forma en que las personas viven, se relaciona con patrones de conducta seleccionados como alternativas disponibles que los individuos prefieren, de acuerdo con la propia capacidad para elegir, influenciada por factores socioeconómicos (OMS, 1986), sociales y culturales, entre otros. En la actualidad, los patrones de comportamiento se han modificado repercutiendo en un estilo de vida (EV) no saludable donde las conductas de riesgo como tabaquismo, consumo de alcohol, exceso de peso, sedentarismo, dieta pobre en granos, frutas y verduras, etc., son responsables de la gran carga mundial de morbilidad de enfermedades crónica degenerativas o sus complicaciones.

En el 2010, la muerte y la carga de enfermedad atribuible a factores de riesgo conductuales y dietéticos, se superpuso con algunos otros agentes como el Índice de Masa Corporal (IMC) alto (indicador de obesidad), y niveles de colesterol y glucosa elevados, asociándose los factores de riesgo a estilos de vida poco saludables (Ezzati, 2013). La carga de enfermedad, que se mide como años de vida ajustados por discapacidad, por sus siglas en inglés DAYLs (Disability-Adjusted Life-Year), relacionada a estilos de vida poco saludable y a sus consecuencias, propicia el doble de años de vida perdidos por discapacidad (6.5), comparado con el Índice de Masa Corporal IMC alto (3.6), la hiperglucemia o el sedentarismo (3.5 y 3.0) (Ezzati, 2013). Todos estos componentes están relacionados con la prevalencia de enfermedades crónico degenerativas, tales como diabetes mellitus, obesidad y sobrepeso, resistencia a la insulina y poliquistosis ovárica. Juntas todas estas patologías, constituyen el llamado Síndrome Metabólico (SM) (INEGI/ Secretaría de salud, 2008; Méndez-Hernández, 2009).

Existen diversos criterios para el diagnóstico de SM, sin embargo en el presente estudio consideramos basarnos en el propuesto por la Federación Internacional de Diabetes (Barquera Cervera, CamposNonato, Rojas \& Rivera, 2010; Echevarría, Hernández, Alcocer, 
Morales \& Vázquez, 2006; Rojas Martínez, Aguilar-Salinas \& JiménezCorona, 2012), debido a que es aceptado por diferentes organizaciones de salud, está actualizado, es adecuado para la población mexicana y propone a la obesidad central o androide como factor de riesgo principal, además de elegir entre otras dos alteraciones metabólicas. El índice de cintura (IC) valora la obesidad central o androide, este tipo de obesidad nos indica la presencia de grasa visceral (Ríos \& Legorreta, 2005). Es cierto que la obesidad incrementa el riesgo de enfermedades crónicas. Sin embargo, estudios recientes muestran que son los pacientes con obesidad visceral los que forman el subgrupo de individuos con las alteraciones más graves del metabolismo. Así, se ha demostrado que la acumulación regional de grasa (determinada por el IC) en los depósitos viscerales es factor predictivo de riesgo cardiovascular más fiable que la cantidad total de grasa corporal e (IMC) (Scarsella, 2003).

La prevalencia del SM aumenta con la severidad de la obesidad hasta alcanzar un 50\% de los jóvenes más obesos, según un estudio realizado con nińos y adolescentes obesos (Ram Weiss et al., 2004, citado en Botero, Salazar \& Cortés, 2006), en donde se dedujo que la prevalencia en pacientes moderadamente obesos era de $38.9 \%$, y en severamente obesos era de $49.7 \%$

En una revisión sistemática de las características del síndrome metabólico en adolescentes de 10 a 19 años, en países en vías de desarrollo, se halló una prevalencia variable del síndrome metabólico (entre 4.2 a 15.4\%), según los criterios del National Cholesterol Education Program (NCEP) - ATP III (Adult Treatment Panel III), y entre 4.5 al 38.7\%, según los criterios de la OMS para la misma población. La hipertrigliceridemia fue el componente reportado con mayor frecuencia (prevalencia variable del 4.9 al 75\%), mientras que la glucemia basal alterada fue el parámetro menos diagnosticado (Hayes Dorado, 2009). La prevalencia de SM es variable según el criterio aplicado. En una población joven europea, se reportó el 5.25\%, según criterios del ATPIII; y 5.28\%, según el ATP-III modificado, elevándose a un $12.64 \%$ cuando se agregó el criterio de Homeostasis Model Assessment (HOMA) como factor de riesgo (Hayes Dorado, 2009). En relación a la frecuen- 
cia del síndrome metabólico en adolescentes de diferentes edades, se ha observado que su incidencia fue el doble en adolescentes de 14 a 18 años, en comparación a niños de 8 a 12 años. Este hallazgo se puede explicar porque las hormonas sexuales y el balance de andrógenos/ estrógenos juegan un rol importante en la modulación de la resistencia a la insulina y del síndrome metabólico.

Respecto a la influencia de los factores ambientales, se ha observado que los asociados al síndrome metabólico en adolescentes son: la rápida transición nutricional, la migración del ámbito rural al urbano, la adopción de un estilo de vida sedentario y la condición materna fetal relacionada.

Hayes Dorado (2009) reportó que en los adolescentes de sexo femenino, el estrato socio económico elevado, los antecedentes de sobrepeso en los padres, los hábitos familiares y el consumo elevado de carbohidratos, constituyeron los factores más influyentes sobre la prevalencia de SM (la prevalencia de SM fue del 6.5\% en la población general y del $45 \%$ en las adolescentes obesas). Otro investigación realizada en Argentina (Predroso et al., 2005), reportó una prevalencia de SM de $4.5 \%$ significativamente mayor en varones que en mujeres (7.5\% frente a $2.5 \% ; p=0,006)$, al igual que en el grupo de 15 a 20 años sobre el de 11 a 14 años. Del total de adolescentes de la muestra un $84,8 \%$ eran sedentarios.

En México, aplicando los criterios propuestos por la OMS sobre el SM, cerca del $10 \%$ de las personas mostraron tolerancia normal a la glucosa, 40\% manifestaron intolerancia a la glucosa, y en la población con diabetes tipo 2, un $90 \%$ podría tener SM (Carrillo Esper, Sánchez Zúñiga \& Argueta Elizondo, 2011). La Encuesta Nacional de Salud 2012 (Gutiérrez et al., 2012) demostró una prevalencia de sobrepeso u obesidad en un 35\% de adolescentes entre 12 y 19 ańos. En 2006 la prevalencia combinada se había registrado en un 33.2\%, lo que reporta un aumento del $5 \%$ en los últimos seis ańos. La proporción de sobrepeso para el 2012 fue más alta en mujeres (23.7\%) que en hombres (19.6\%). En el caso de la obesidad, los datos revelaron que el porcentaje de adolescentes del sexo masculino con obesidad fue mayor 
(14.5\%) que en las de sexo femenino (12.1\%). En la población mayor de 20 años, se registró un aumento de IMC acelerado de 1988 a 2012. Asimismo, el sobrepeso en mujeres de 20 a 49 años se incrementó de 25 a $35.3 \%$, mientras que la obesidad pasó de un 9.5 a un 35.2\%; aunque en los últimos 6 ańos (2006 a 2012) la prevalencia agregada de sobrepeso y obesidad en mujeres adultas mostró una reducción en la velocidad de aumento cercana al 2\% anual (Gutiérrez et al., 2012).

La obesidad en la mujer tiene matices peculiares que comienzan a ser comprendidos. La mayor prevalencia de obesidad en este grupo es producto de características biológicas y psicológicas, así como de situaciones sociales. En comparación con los hombres, el mayor porcentaje de masa grasa en las mujeres determina un gasto energético menor por kilogramo de peso, lo que hace más difícil perder el sobrepeso ganado. Los embarazos, la ansiedad y la depresión, frecuentes en mujeres obesas, son a la vez causa y consecuencia de la misma.

El ambiente «obesogénico» puede tener mayor efecto en las mujeres porque las porciones que ingieren son habitualmente superiores a sus necesidades, ya que sus requerimientos energéticos son menores que los de los hombres por su fenotipo y su nivel de actividad. Respecto a las consecuencias de la obesidad y del síndrome metabólico, se ha concluido en algunos análisis que las repercusiones psicosociales y económicas negativas de la obesidad, son mayores entre las mujeres (García-García et al., 2009).

En México, el desarrollo económico, la urbanización y la influencia tecnológica y cultural presente también en países industrializados, ha influido y modificado algunos estilos de vida, contribuyendo a una marcada disminución en la actividad física. Solo 35\% de las personas entre 10 y 19 ańos de edad son activas, más de la mitad de estos adolescentes pasan 14 horas semanales o más frente a la televisión, y otro $25 \%$ pasa hasta tres horas diarias en promedio. Entre los adolescentes y adultos jóvenes (de 12 a 29 ańos de edad) tan solo $40 \%$ practica algún tipo de actividad física, siendo el sedentarismo es mayor en mujeres que entre hombres (Leija-Alva et al., 2011). 
Las enfermedades crónicas degenerativas asociadas a este estilo de vida han hecho evidente el papel del ambiente, de las conductas de la sociedad y de los individuos en su desarrollo (García-García et al., 2009). La elevada prevalencia de estilos de vida poco saludables, asociada en algunos casos a enfermedades crónico degenerativas incipientes, se ha relacionado a indicadores emocionales que repercuten en el aprovechamiento académico (Álvarez Gasca, Ríos, Rodríguez \& Lozano, 2008). El SM es pobremente diagnosticado y mal tratado, por lo que es importante atender a las alteraciones metabólicas, hemodinámicas, hemorreológicas y sus complicaciones, ya que son fundamentales para evitar la progresión de la enfermedad. Sin embargo la clave para la disminución de su prevalencia y morbimortalidad, está en la prevención y el diagnóstico oportuno. Incrementar la autoconciencia de riesgo percibido desde edades tempranas puede ser una alternativa eficiente para apoyar las campañas educativas a la población joven.

En relación a los antecedentes y tomando en cuenta que la población estudiada estuvo compuesta por futuros promotores de salud, el objetivo de esta investigación fue analizar la relación entre estilo de vida y síndrome metabólico en estudiantes de carreras relacionadas con la salud, así como las posibles diferencias entre hombres y mujeres. De igual manera, se considera importante la implementación de programas sobre estilos de vida saludables que fomenten la cultura de actividad física y ejercicio regular, manejo del estrés y emociones, calidad de sueño y consumo de alimentos y bebidas saludable, con el propósito de procurar la prevención o rehabilitación de trastornos metabólicos que permita a los estudiantes lograr un desarrollo académico y eficiencia terminal adecuados.

\section{Método}

\section{Participantes}

En el estudio participaron 972 estudiantes recién ingresados a carreras relacionadas con la salud que se imparten en la Facultad de 
Estudios Superiores Iztacala (FESI) de la Universidad Nacional Autónoma de México (UNAM). La edad promedio de los estudiantes fue de 18.2 años $(D T=1.7)$, de los cuáles $655(67.4 \%)$ fueron mujeres y 317 (32.6\%) hombres. El muestreo fue probabilístico, estratificado entre las carreras de medicina, odontología, psicología, enfermería, optometría y biología.

\section{Medidas e instrumentos de medida}

La evaluación se realizó mediante la aplicación de un cuestionario, mediciones antropométricas, presión arterial y pruebas sanguíneas.

La valoración del Estilo de Vida (EV) y su relación que tiene con la salud se realizó con la escala FANTASTIC (Wilson \& Ciliska, 1984) versión mexicana (López-Carmona, Rodríguez-Moctezuma, Munguía-Miranda, Hernández-Santiago \& Casas-de la Torre, 2000). Este instrumento consta de 25 ítems cerrados que exploran nueve dominios sobre componentes físicos, psicológicos y sociales del EV: familia-amigos, actividad física, nutrición, tabaco-toxinas, alcohol, sueño-cinturón de seguridad-estrés, tipo de personalidad, interior (ansiedad, preocupación, depresión) y carrera-labores. Se califica por medio de una escala tipo Likert, según los niveles: excelente, bueno, regular, malo y en peligro (Rodríguez, López, Murguía, Hernández \& Martínez, 2003).

\section{Criterios diagnósticos}

- La obesidad central se determinó según la circunferencia de cintura $\geq 90 \mathrm{~cm}$ para hombres $\mathrm{y} \geq 80 \mathrm{~cm}$ para mujeres.

- El Síndrome metabólico se determinó siguiendo los criterios de la Federación Internacional de Diabetes (Barquera Cervera et al., 2010; Echevarría et al., 2006; Rojas Martínez et al., 2012), según la presencia de la obesidad central más dos de los factores siguientes: glucosa en ayuno $\geq 100 \mathrm{mg} / \mathrm{dl}$., triglicéridos $\geq 150 \mathrm{mg} / \mathrm{dl}$., colesterol $\mathrm{HDL}<40 \mathrm{mg} / \mathrm{dl}$ en hombres o $<50$ en mujeres; presión arterial $\geq 130 / 85 \mathrm{mmHg}$. 


\section{Procedimiento}

Los estudiantes fueron informados del objetivo y de la importancia del estudio. Luego de recogerse el consentimiento informado, se dieron las indicaciones de manera verbal y escrita para que asistieran a la evaluación en fechas determinadas con el permiso de sus profesores. Un laboratorio clínico realizó las pruebas bioquímicas para valorar el estado metabólico y la presencia o no de síndrome metabólico en los participantes. Luego se evaluó la presión arterial y se tomaron medidas antropométricas de peso, talla y perímetro de cintura para determinar la presencia de obesidad central. Finalmente, se aplicó el cuestionario Fantastic. El proceso de evaluación duró cuatro semanas. Posteriormente, se convocó a los participantes para ofrecerles los resultados de su evaluación, así como el apoyo correspondiente. Los datos obtenidos se organizaron, clasificaron y se analizaron con el paquete estadístico Minitab versión 15. Luego se determinó la relación entre síndrome metabólico y el estilo de vida, obesidad y género, para el análisis y la relación de variables, se aplicó la prueba de $X^{2}$.

\section{Resultados}

Se presentan los resultados de hombres y mujeres del estilo de vida de los estudiantes en sus diferentes niveles, la frecuencia de obesidad, síndrome metabólico y la relación entre dichas variables. En la población total, el estilo de vida que predominó en más de la mitad de los estudiantes, fue el nivel bueno con 535 casos (55\%), siguiendo el regular con 184 casos (18.93\%), excelente en tercer lugar con 181 casos (18.62\%), malo con 70 casos (7.2\%) y en peligro solo 2 casos ( $0.21 \%)$. En la Figura 1 se muestra que, del total de participantes, el EV bueno fue muy similar para ambos sexos, siendo este nivel el que predominó; el segundo lugar lo ocupó el EV regular, donde las mujeres puntuaron un poco arriba de los hombres, luego se encontraba el EV excelente, en donde los hombres calificaron por arriba de las mujeres, mientras que el EV malo se hallaba en cuarto lugar, siendo superior en el caso de las mujeres. 


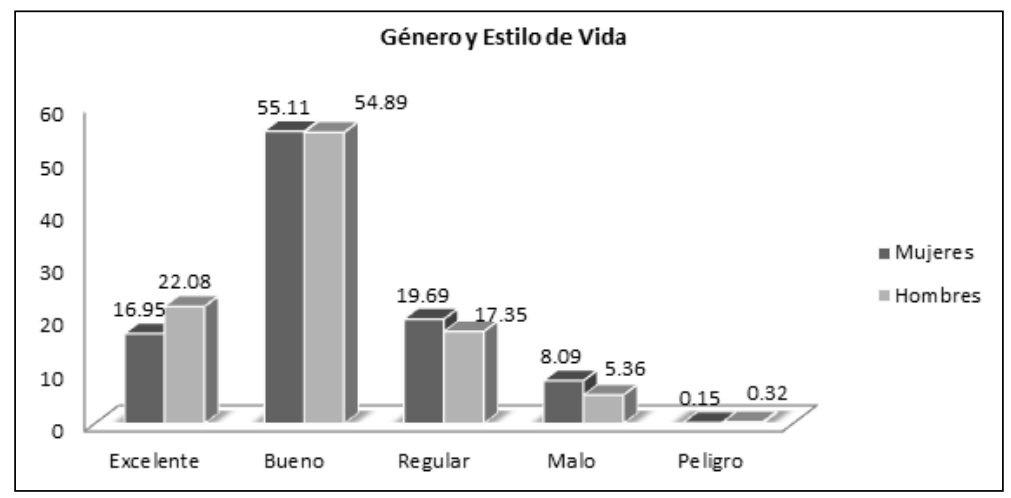

Figura 1. Niveles de estilo de vida en porcentajes por sexo en estudiantes universitarios de primer ingreso

Se diagnosticó SM en 45 estudiantes (4.63\%), de los cuales 8 casos fueron hombres $(0.82 \%)$ y 37 mujeres $(3.81 \%)$. Del $95.37 \%$ que no presentaron SM, 309 casos (31.79\%) fueron hombres y 618 (63.58\%) mujeres. Aparentemente el porcentaje es bajo, pero se puede notar que 45 estudiantes presentaron esta enfermedad metabólica a muy temprana edad y que las mujeres superaron en mucho a los hombres, lo cual es reflejo de la obesidad central. En la Figura 2, se muestra que el SM se presentó en mayor proporción en las mujeres con respecto a los hombres, y la prueba de $X^{2}$ mostró una relación significativa entre género y SM $(p<.05)$. 


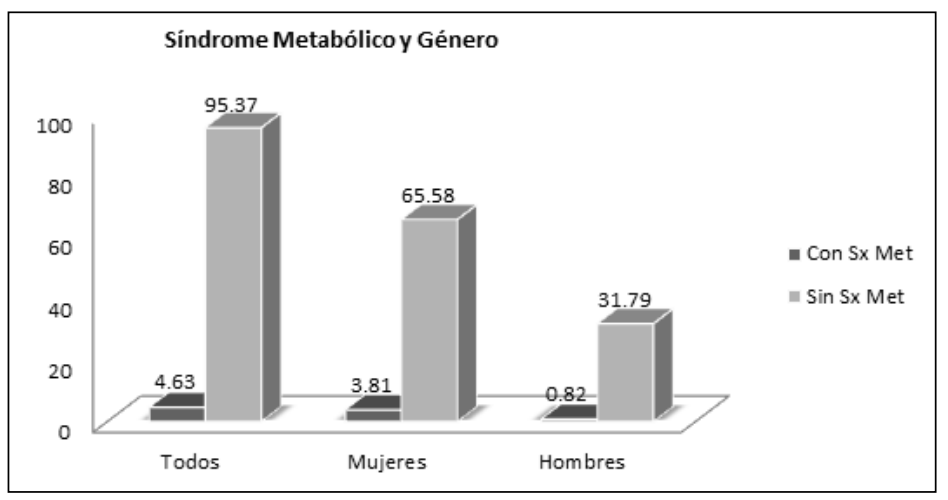

Figura 2. Distribución porcentual del síndrome metabólico por sexo en estudiantes universitarios de primer ingreso.

El 36.65\% de los estudiantes obtuvieron indicadores que señalaban obesidad de tipo central, presentando las mujeres (26.5\%) más del doble de casos que los hombres (10.14\%). Los resultados mostraron una relación significativa entre género y obesidad $(p<.05)$ (ver Figura 3).

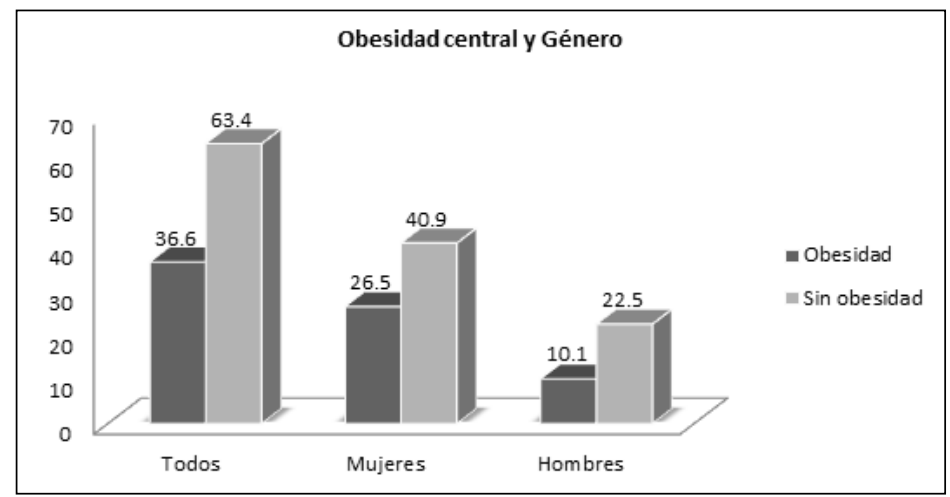

Figura 3. Distribución porcentual de obesidad central en estudiantes universitarios de primer ingreso por sexo. 
En la figura 4 se muestra el estilo de vida en sus diferentes niveles y su relación con el síndrome metabólico. Se puede apreciar que el EV que se presentó más frecuentemente fue el malo, seguido por el regular. Sin embargo, hubo una baja frecuencia de EV en peligro.

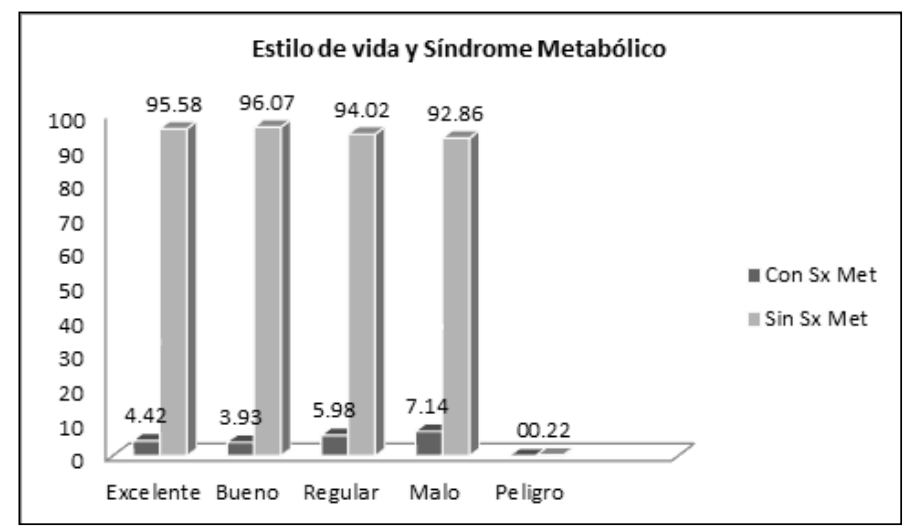

Figura 4. Síndrome metabólico por nivel de estilo de vida en estudiantes universitarios de primer ingreso.

\section{Discusión}

En términos generales se puede afirmar que el estilo de vida encontrado resultó ser similar entre hombres y mujeres, lo cual se explica debido a que en la actualidad los patrones de comportamiento han modificado el estilo de vida de las personas con conductas poco saludables como dietas bajas en frutas, verduras y sedentarismo, entre otras variables, tal como lo menciona Ezzati y Riboli (2013), y MéndezHernández (2009). Esto favorece la Obesidad, el SM y estilos de vida regulares, malos y hasta peligrosos como los que se encontraron en el presente estudio.

Por otro lado, la prevalencia de SM es variable de acuerdo a los criterios diagnósticos. Coincidimos con lo encontrado por Hayes-Dorado 
(2009) en una revisión sistemática en la que se realizaron diversos estudios en adolescentes de 10 a 19 años en países en vías de desarrollo, donde el diagnóstico de SM de acuerdo al criterio del ATP III fue del $4.2 \%$ a $15.4 \%$, mientras que según la OMS sería entre $4.5 \%$ a $38.7 \%$. Lo hallado en el presente estudio, según la Federación Internacional de Diabetes, fue de $4.63 \%$. Estos resultados son coherentes con los anteriores, a excepción del hecho de que la frecuencia de SM resultara ser mucho mayor en mujeres que en hombres, lo cual difiere del estudio de Pedroso y colaboradores (2005) realizado en adolescentes argentinos de edades similares. Sin embargo, esto difiere de lo encontrado por Cardoso y colaboradores (2010), quienes hallaron frecuencias casi iguales de SM entre hombres (11.5\%) y mujeres (13.5\%) en una muestra conformada por adolescentes mexicanos.

Según datos de la Encuesta Nacional de Salud 2012, en México el $35 \%$ de adolescentes entre 12 y 19 años presentó obesidad, siendo este porcentaje mayor en mujeres (23.7\%) que en hombres (19.6\%). Esto coincide con lo hallado en esta investigación, en donde del total de la muestra el $36.65 \%$ presentó obesidad, encontrándose más en mujeres (26.5\%) que en hombres (10.14\%), lo cual refleja una parte de la situación de salud en nuestro país.

A partir de todo lo expuesto, se concluye que sí hubo diferencias entre hombres y mujeres, ya que la relación entre género y obesidad resultó significativa, al igual que la relación entre género y síndrome metabólico. Por todo esto, es importante considerar la elevada frecuencia de obesidad, ya que es un factor de riesgo para enfermedades crónico degenerativas como el cáncer, entre otras. Asimismo, estas variables influyen en la salud psicológica y social, sobre todo en las mujeres, por lo que la presencia de síndrome metabólico, aunque en menor frecuencia, es una señal de alarma debido a la edad de la población. Se sugiere la implementación de programas para prevenir y rehabilitar conductas de riesgo relacionadas a la obesidad y al síndrome metabólico. 


\section{Referencias}

Álvarez Gasca, M.A., Ríos S., Rodríguez, S. \& Lozano, T. (2008). Estilo y calidad de vida en estudiantes universitarios con enfermedad crónica. Alternativas en Psicología, 8(17), 41-49.

Barquera Cervera, S., Campos-Nonato, I., Rojas, R \& Rivera, J. (2010).

Obesidad en México: epidemiología y políticas de salud para su control y prevención. Gaceta Médica de México, 146, 397-407.

Botero, J. C., Salazar, D. \& Cortés O. (2006). Síndrome metabólico y riesgo cardiovascular. Revista CES Medicina, 20(2), 73-81.

Cardoso-Saldaña G.C., Yamamoto-Kimura L., Medina-Urrutia A., Posadas-Sánchez R., Caracas-Portilla N.A \& Posadas-Romero C. (2010). Exceso de peso y síndrome metabólico en adolescentes de la Ciudad de México. Obesity or overweight and metabolic syndrome in Mexico City teenagers. Archivos de Cardiología de México, 80(1).

Carrillo-Esper, R., Sánchez-Zúńiga A.J. \& Argueta Elizondo S. (2011). Sindrome Metabólico. Recuperado de www.ejournal.unam.mx/ rfm/no49-3/RFM49305.pdf.

Echavarría, P.M., Hernández, L.A., Alcocer, G.M., Morales, F.H. \& Vázquez, M.A. (2006). Síndrome metabólico en adultos de 20 a 40 años en una comunidad rural mexicana. Revista Médica del IMSS, 44(4), 329-35.

Ezzati, M. \& Riboli, E.(2013). Behavioral and dietary risk factors for noncommunicable diseases. The New England Journal of Medicine, 369, 954-64. doi:101056/NEJMra1203528.

García-García, E., De la Llata, M., Kaufer-Horwitz, M., Tausé-Luna, M., Calzada-León, R., Vázquez-Velásquez, V.,... Sotelo-Morales, J. (2009). La obesidad y el síndrome metabólico como problema de salud pública. Una reflexión. Segunda parte. Salud Mental, 32(1), Recuperado de http://www.scielo.org.mx/scielo. php?script $=$ sci_arttext\&pid=S0185-33252008000600010

Hayes-Dorado, J.P. (2009). Síndrome metabólico en niños y adolescentes. Rev. bol. ped., 48(2), 96-100. 
INEGI/Secretaría de salud. (2008). Dirección General de Información en salud. CONAPO. México. Recuperado de http://sinais. salud.gob.mx/mortalidad

Leija-Alva G., Aguilera-Sosa V.R., Lara-Padilla E., Rodríguez-Choreño J.D., Trejo-Martínez J.I. \& López de la Rosa, M.R. (2011). Diferencias en la modificación de hábitos, pensamientos y actitudes relacionados con la obesidad entre dos distintos tratamientos en mujeres adultas. Revista Latinoamericana de Medicina Conductual, 1(22), 19-28. doi:10.5461/rlmc.v1.i2.20964.

Gutiérrez, J.P., Rivera-Dommarco, J., Shamah-Levy, T., VillalpandoHernández S, Franco A, Cuevas-Nasu, L., Romero-Martínez, M. \& Hernández-Ávila, M. (2012). Encuesta Nacional de Saludy Nutrición. Resultados Nacionales. Cuernavaca, México: Instituto Nacional de Salud Pública (MX).

López-Carmona, J.M., Rodríguez-Moctezuma, R., Munguía- Miranda, C., Hernández-Santiago, J.I. \& Casas-de la Torre, E. (2000). Validez y fiabilidad del instrumento FANTASTIC para medir el estilo de vida en pacientes mexicanos con hipertensión arterial. Atención Primaria. 26(8), 542-549.

Pedroso,W., Castillo Rascón, M., Bonneau, G., Ibáńez de Pianesi, M., Castro Olivera, C. \& Jiménez de Aragón,S. (2005). Síndrome metabólico y factores de riesgo asociados con el estilo de vida de adolescentes de una ciudad de Argentina. Rev. Panamericana de Salud Pública. 24(3), 149-160.

Organización Mundial de la Salud [OMS] (1986). Carta de Otawa para la promoción de la salud. Recuperado de http://www.paho. $\mathrm{org} / \mathrm{spanish} / \mathrm{hpp} /$ otawachartersp.pdf

Rojas Martínez, R., Aguilar-Salinas, C. \& Jiménez-Corona, A. (2012). Puntos de corte óptimos para la detección de diabetes de tipo 2, hipertensión y síndrome metabólico no diagnosticados en adultos mexicanos. Salud Pública de México, 54(1).

Rios, L. \& Legorreta, J. (2005). Distribución de grasa corporal en diabéticos tipo 2, como factor de riesgo cardiovascular. Revista Médica del IMSS, 43(3), 199-204. 
Rodríguez, M.R., López, C.J., Murguía, M.C., Hernández, S.J. \& Martínez, B.M. (2003). Validez y consistencia del Instrumento FANTASTIC, versión mexicana. Revista Médica del IMSS, 41(3), 211-220.

Scarsella, C. (2003). Tratamiento de la obesidad: necesidad de centrar la atención en los pacientes de alto riesgo caracterizados por la obesidad abdominal. Cadernos de Saúde Pública, 19(1), 7-19.

Secretaria de Salud (2008). Principales causas de mortalidad general. Recuperado de http://sinais.salud.gob.mx/mortalidad

Wilson, D.M. \& Ciliska, D. (1984). Life-style assessement: Development end use of the FANTASTIC checlist. Canadian Family Physician, 30, 1527-1532.

Recibido: 20 de noviembre, 2013

Aceptado: 03 de diciembre, 2013 


\title{
Anexo
}

\section{A Consentimiento informado}

\author{
UNIVERSIDAD NACIONAL AUTÓNOMA DE MÉXICO \\ FACULTAD DE ESTUDIOS SUPERIORES IZTACALA
}

Grupo Multidisciplinario de investigación en Salud y Rendimiento Académico GMISARA

\section{Carta de consentimiento informado}

Nombre del alumno:

Si el alumno es menor de edad:

Nombre del tutor: Parentesco:

Edad Correo electrónico

Declaro que se me ha explicado la conveniencia de responder a una batería de evaluación psicológica y a proceder a tomar una muestra sanguínea de una vena periférica, con la finalidad de valorar mi estado de salud. Estoy enterad@de las posibles complicaciones menores, que resulten del segundo procedimiento y que incluyen malestar en el sitio de punción y raramente una pequeña zona de equimosis (moretón).

Estoy informad@que el equipo para la toma de sangre es estéril, desechable y será abierto en mi presencia. También he sido enterad@ de que los resultados de ambos procedimientos serán manejados de forma ética y con discreción, precisando que únicamente serán entregados de manera personal a cada uno de los interesados que lo soliciten. Asimismo manifiesto estar enterad@ de que las muestras biológicas no serán utilizadas para analizar indicadores de VIH, tampoco embarazo, ni consumo de drogas o alcohol.

Consiento que si se detecta alguna alteración en los estudios propuestos, seré convocad@a recibir una amplia información de los resultados; así como recibir la asesoría para precisar la alteración, el seguimiento y sus posibilidades de manejo. 
Declaro que he comprendido los puntos antes señalados y me reservo expresamente el derecho de revocar mi consentimiento, antes o después de tomar las muestras, sin que medie explicación alguna.

Los Reyes Iztacala, Tlalnepantla México a de de

Nombre y firma del Alumno Nombre y firma del tutor (Sólo si es menor de 18 años) 